\title{
PTSS IN FATHERS OF VLBW INFANTS TWO TO FOUR YEARS POSTPARTUM:
}

\section{A PILOT STUDY}

Amy L Alexander ${ }^{1}$, Paul Clarke ${ }^{2,3}$, Imogen Rushworth ${ }^{1} \&$ Kiki Mastroyannopoulou ${ }^{1}$

${ }^{1}$ University of East Anglia, Department of Clinical Psychology, Norwich Medical School, Norwich, UK. ${ }^{2}$ Neonatal Unit, Norfolk and Norwich University Hospitals NHS Foundation Trust, Norwich, UK. ${ }^{3}$ Department of Medicine, Norwich Medical School, University of East Anglia, Norwich, UK.

Corresponding author: A. Alexander, CAMHS, The Newtown Centre, Nursery Road, Huntingdon, PE29 3RJ, UK. Tel +44 7809 148048. Email amy@alexanders.me.uk; amy.alexander@cpft.nhs.uk

Prior presentation: This study was presented in poster form at the $6^{\text {th }}$ Congress of the European Academy of Paediatric Societies, Geneva, Switzerland, October 2016.

\section{Acknowledgements}

The authors sincerely thank Dr Susanne Åhlund for kindly sharing her maternal PTSD dataset from her previous study. Amy L. Alexander acknowledges the kind funding received from Chiesi Uk Ltd and Danone Ltd which covered the postage and stationery costs of the study questionnaires. These companies otherwise had no input into or involvement with the study. All other study costs were funded internally by the UEA Department of Clinical Psychology and by the Norfolk and Norwich University Hospital Neonatal Unit Research Fund. Amy L Alexander would also like to thank Jill Tinsey, Laura Collins and Tracy Oliver for their help with searching the maternity and neonatal databases and to Dave Peck for statistics advice. 


\begin{abstract}
Aim: To conduct a pilot study to compare fathers of very low birth weight (VLBW; $<1500$ grams) infants and of term infants at two to four years postpartum for self-reported post-traumatic stress symptoms (PTSS) related to the previous birth of their baby, and for depression, and anxiety. The prevalence of paternal PTSS was compared with that of a historical cohort of mothers of VLBW infants followed up at a similar postnatal age.
\end{abstract}

Methods: 26 fathers of VLBW infants and 22 fathers of term infants completed four validated psychological questionnaires at two to four years postpartum. Results: PTSS levels were significantly higher in fathers of VLBW infants than in fathers of term infants, and PTSS levels did not differ significantly between fathers and the mothers of VLBW infants of an historical cohort. There was a significant association between perceived levels of social support and PTSS severity in fathers of VLBW infants, but not for their anxiety or depression severity. Conclusion: PTSS directly related to the birth of their VLBW infants are present in fathers at two to four years postpartum and occur at similar levels to those found in mothers of VLBW infants. Routine screening for PTSS in fathers of VLBW infants is important and targeted psychological support should be offered. Larger scale studies in the area are needed to elucidate further information about PTSS in fathers postpartum.

Keywords: Intensive care, postnatal, premature, preterm, trauma

\title{
Implications for Impact Statement
}

Fathers of very low birth weight infants (VLBW) infants experience significantly greater levels of post-traumatic stress symptoms (PTSS) than fathers of term infants, two to four years postpartum. Fathers of VLBW infants experience similar high levels of PTSS to mothers of VLBW infants at two to four years postpartum. These findings highlight the 
importance of screening fathers for such difficulties and the need to implement psychological support for them.

\section{Introduction}

Following birth, very low birth weight (VLBW; <1500g) infants require care within Neonatal Intensive Care Units (NICUs). In 2013, $1 \%$ of all babies born had birth weights $<1500$ grams (Office for National Statistics, 2014). Most (80-90\%) VLBW infants are born prematurely, at between 24 and 32 weeks gestational age (Shah et al., 2016). The birth and subsequent NICU experience can often be extremely traumatic for parents (Elklit, Hartvig, \& Christiansen, 2007; Lefkowitz, Baxt, \& Evans, 2010). Traumatic events can lead to posttraumatic stress disorder (PTSD). Post-traumatic stress symptoms (PTSS) refer to when individuals develop some, but not all, symptoms of PTSD and thus do not meet diagnostic criteria. PTSS are higher in mothers of high-risk infants, including mothers of preterm, VLBW and sick term infants compared with in mothers of healthy full-term infants (Åhlund, Clarke, Hill, \& Thalange, 2009; Ionio et al., 2016).

Witnessing a traumatic event is included in the criteria for PTSD in the Diagnostic and Statistical Manual of Mental Disorders Fifth Edition (DSM-5; American Psychiatric Association [APA], 2013). Men attending childbirth may experience many hours of significant arousal, with vivid images of a potentially life-threatening situation affecting their partner and baby. Fathers may experience PTSD after witnessing a difficult birth at term (Ayers, Wright, \& Wells, 2007). The limited research exploring PTSS in fathers of premature and VLBW infants has reported widely varying rates of PTSS, ranging from 1.2\% to $65.8 \%$ (Binder, Zeltzer, Simmons, Mirocha, \& Pandya, 2011; Ghorbani, Dolatian, Shams, \& Alavi-Majd, 2014; Koliouli, Gaudron, \& Raynaud, 2016; Lefkowitz et al., 2010; Mehler et al., 2014; Shaw et al., 2009). Only one study specifically investigated presence of PTSD in fathers of VLBW infants and found no evidence of fathers meeting criteria (Elklit et al., 
2007). The majority of studies focused on immediate psychological impact, up to six months postpartum. The sole study assessing PTSD beyond six months recruited parents up to threeand-a-half years postpartum, though the majority of the participants were recruited at six to 12 months (Elklit et al., 2007).

Depression and anxiety disorders are commonly comorbid with PTSD (Grinage, 2003), and in women comorbid depression with postnatal PTSD is high (Stramrood et al., 2011; White, Matthey, Boyd, \& Barnett, 2006). Paternal PTSS and potential comorbid anxiety and depression following the birth of a VLBW infant may affect attachment and may disrupt father-infant bond development, potentially impacting the child's psychological, social and cognitive development (All Party Parliamentary Group for Conception to Age 2: The First 1001 Days, 2015). Nurturing the emotional well-being of parents in the NICU is important, both to optimise their long-term emotional well-being and to aid the relationship with their baby, which can positively impact the infant's growth and development (Hynan et al., 2015).

There is a need within the NICU environment for normalisation and validation of emotional distress rather than labelling this as pathological (Hynan, Mounts, \& Vanderbilt, 2013; Hynan et al., 2015). Screening can assist in this process of normalisation (Hynan et al., 2013). Hynan et al. (2013) recommend screening both parents at the start of a NICU admission, that this should ideally also take account of physical and mental health histories, and to re-screen prior to discharge for those staying for more than 10-14 days and at followup clinics.

The purpose of our study was to expand on the limited research on PTSS in fathers of VLBW infants postpartum and to explore potential additional factors such as depression, anxiety and social support. Recommendations from Phares, Lopez, Fields, Kamboukos, \& 
Duhig (2005) are that when a characteristic in mothers is investigated it should also be investigated in fathers. Åhlund et al. (2009) showed that mothers had a high prevalence of PTSS two to three years following the birth of their VLBW infant, but fathers were not included. Using similar methodology, the present study therefore sought to investigate whether fathers of VLBW infants may also have high levels of PTSS after a comparable duration. We aimed to answer the following research questions: i) is there a difference in levels of PTSS, anxiety and depression in fathers of VLBW infants compared with fathers of term-born infants at two to four years postpartum? ii) What is the impact of perceived social support on severity of PTSS, anxiety and depression? iii) How does the prevalence of PTSS in fathers of VLBW infants at two to four years postpartum compare with that seen in mothers of VLBW infants two to three years postpartum?

\section{Methods}

\section{Participants}

The term "father" referred to the male who considered themselves the father at the time of the birth of the child. Inclusion criteria for fathers of VLBW infants were admission of a live-born infant of birth weight $<1500 \mathrm{~g}$ to the NICU of our regional hospital. To reduce the potential confounding factor of traumatic term birth, fathers of term infants were included only if their infant did not require NICU admission and was born at $>37$ weeks' gestation via unassisted vaginal delivery. Fathers were excluded if they were under the age of 18 , were non-English-speaking, or if their infant had been adopted or died. Participant baseline characteristics are shown in Table 1. In the VLBW group, there were 26 fathers of 17 (63\%) male and $10(37 \%)$ female infants; one was a father of twins. In the term group there were 21 fathers of $13(59 \%)$ male and $9(41 \%)$ female infants, and none was a father of multiples. The median birthweight of VLBW infants was 1060g (IQR: 769-1351g) and of term infants 
was 3593g (IQR: 3288-3898g) and median gestational ages were 28 weeks (IQR $=25-31$ weeks) and 40 weeks (IQR $=39-41$ weeks) respectively.

\section{Design}

All VLBW infants born between June 2011 and June 2013 were identified through the neonatal electronic patient record (BadgerNet Neonatal, Clevermed Ltd, UK). Study invite letters were sent from our hospital to fathers of VLBW infants who met inclusion criteria. Study invitations were also sent to fathers of term infants matched by date of birth but not gender. In total, 166 fathers in both VLBW and term groups were initially invited to participate (Figure 1). Nine additional fathers of VLBW infants were recruited through a two-year follow-up clinic; their infants had been born between June and November 2013; control-group matching was not done for these cases. Questionnaire packs with stampedaddressed envelopes for return were sent to fathers who consented to receive them. A total of 31 fathers of VLBW infants and 26 fathers of term infants consented to receive questionnaire packs. Reminder letters were used to aid recruitment. Recruitment flow diagram is shown in Figure 1.

The decision to conduct this study two to four years postpartum was taken because, firstly, the limited research that has been conducted on the psychological reaction of fathers following the birth of a VLBW infants has focussed on the short-term effects (e.g. Lefkowitz et al., 2010; Shaw et al., 2009) and on acute hospital rather than community experiences (Deeney, Lohan, Parkes, \& Spence, 2009). Secondly, the aim was to compare the data from this study with that found in mothers by Åhlund et al. (2009) which used a two to three years postpartum time-frame. The current study extended this period slightly to aid recruitment. By recruiting from a similar time period, data from the two studies could be compared more meaningfully. Finally, some of the literature on postnatal mental health problems in fathers 
indicates that they may develop later in fathers (e.g. Paulson \& Bazemore, 2010; Matthey, Barnett, Ungerer, \& Waters, 2000).

\section{Measures}

We administered four validated self-report questionnaires to fathers:

1. The Impact of Events Scale-Revised (IES-R; Weiss \& Marmar, 1997) measures PTSS using a 22-item inventory to assess subjective distress and traumatic symptoms following a specific life event. Fathers were asked to indicate how distressing each item had been for them in the previous seven days on a five-point Likert scale ranging from 0 ("not at all") to 4 ("extremely"). The life event specified was the birth of the father's index child and their experience of the hospital care given in the period between this infant's birth and discharge, with questionnaires for VLBW fathers specifically referencing the NICU. This questionnaire assesses how much memories and associated distress of a life event is currently affecting respondents. The total possible score is 88 , scores of 33 or above are considered to indicate a probable PSTD diagnosis (Creamer, Bell, \& Failla, 2003), scores above 24 indicate PTSD is a "clinical concern" (Asukai et al., 2002). Cronbach's alpha values for the total scale and subscales were excellent, suggesting high internal consistency: total scale $\alpha=.96$; intrusion subscale $\alpha=.93$; avoidance subscale $\alpha=.94$; hyperarousal subscale $\alpha=.85$.

2. The ENRICHD Social Support Inventory (ESSI; Mitchell et al., 2003) measures remembered perceived social support at the time of the birth and hospitalisation of the fathers' index infants. Seven items assessed four attributes of social support: emotional, instrumental, informational, and appraisal. Participants were asked to indicate how closely statements described their social support situation at the time of the birth of their index child on a five-point Likert scale ranging from 0 ("none of the time") to 5 ("all of the time") for six items. The final item asked about the presence of a spouse/partner, with a score of 4 given for a positive response and 0 for negative. Higher scores indicate higher social support with a 
possible total score of 4 . This questionnaire did require fathers to recall the situation from the time of the birth and thus may be subject to recall bias. Higher scores indicate greater social support. Cronbach's $\alpha=.88$.

3. The Patient Health Questionnaire-9 (PHQ-9; Kroenke \& Spitzer, 2002) to measure current depression symptoms. Nine items screen, diagnose, monitor and measure the severity of depression; participants indicate how much each item has bothered them in the previous two weeks on a four-point Likert scale ranging from 0 ("not at all”) to 3 (“nearly every day"). The total possible score is 27 , with scores of five to nine indicating mild depression, 10 to 14 indicating moderate, 15 to 19 being moderately-severe, and over 20 indicating severe depression (Kroenke \& Spitzer, 2002). Cronbach's $\alpha=.83$.

\section{The Generalised Anxiety Disorder Seven Item Scale (GAD-7; Spitzer, Kroenke,} Williams, \& Löwe, 2006) to measure current anxiety symptoms. Participants indicate how much each of seven items has bothered them in the previous two weeks on a four-point Likert scale ranging from 0 ("not at all") to 3 ("nearly every day"). The total possible score is 21 , with scores of five to nine indicating mild anxiety, 10 to 14 indicating moderate and over 15 indicating severe anxiety (Spitzer et al., 2006). Cronbach's $\alpha=.81$.

\section{Comparison with Maternal Data}

Maternal comparison data at two to three years post-partum were from the dataset of Åhlund et al. (2009), with permission.

\section{Statistical Analysis}

The non-parametric Mann-Whitney U test was used to compare medians of the two groups because the data were skewed and violated parametric $t$-test assumptions of normality. Data were analysed using SPSS (IBM, Version 22). Bonferroni adjustment was applied to reduce the likelihood of Type I errors when exploring secondary research questions. Thus, when exploring differences between reported anxiety and depression levels in the two groups the 
adjusted alpha was $.05 / 2=.025$. Bonferroni adjustment was also applied when exploring effect of demographic variables on reported PTSS levels. Variables explored were: age of infant, birth weight, gestational age, length of hospital stay, and lengths of stay in the different categories of care provided in UK NICUs (British Association of Perinatal Medicine, 2011) - intensive care, high-dependency care, and special care. Kendall's Tau ( $\tau$ ) explored the associations statistically; a Bonferroni correction was applied (adjusted alpha: $.05 / 7=.007)$.

\section{Ethics}

This study had prior ethical approval from the London-Fulham Research Ethics Committee (REC ref. 15/LO/0947).

\section{Results}

Overall, 26 fathers of VLBW infants and 22 fathers of term infants returned completed questionnaires. These were completed at a mean postpartum age of 3.4 years ( $S D$ $=0.72)$ by fathers of VLBW infants and 3.76 years $(S D=0.69)$ by fathers of term infants.

\section{PTSS}

The mean total IES-R (Weiss \& Marmar, 1997) score for fathers of VLBW infants was $18.1(S D=18.6)$ versus $2.37(S D=4.72)$ for fathers of term infants. Table 2 presents median (IQR) IES-R scores for the two groups. In the VLBW group two fathers (8\%) scored $\geq 24$, indicating that PTSD was a current clinical concern for them, and 5 fathers (19\%) scored $\geq 33$, indicating a probable PTSD diagnosis. No father in the term group scored $>24$; the highest score was 21. Fathers of VLBW infants had significantly higher scores on the IES-R total scale (median $=13$ ) than fathers of term infants (median $=1), U=80.0, z=-4.31$, $p<.001$. Fathers of VLBW infants scored significantly higher on the intrusion $(U=99.5, z=$ 
-3.94, $p<.001)$, avoidance $(U=103, z=-4.09, p<.001)$ and hyperarousal subscales $(U=185$, $z=-2.45, p=.014)$

Exploring the effect of demographic variables. No correlations were significant with an adjusted alpha criterion of .007 . However the correlation between IES-R scores and age was, $\tau=-.37, n=26, p=.008$, suggesting that fathers of VLBW infants may report less PTSS with increasing time interval since birth.

\section{Anxiety and Depression}

The mean total GAD-7 (Spitzer et al., 2006) score for fathers of VLBW infants was $3.35(S D=3.58)$ versus $4.32(S D=3.37)$ for fathers of term infants. Anxiety levels in VLBW fathers (median $=2$ ) also did not differ significantly from those reported by term fathers (median $=4$ ) at 2-4 years postpartum, $U=228, z=-1.22, p=.23$. The mean total PHQ-9 (Kroenke \& Spitzer, 2002) score for fathers of VLBW infants was $2.16(S D=2.21)$ versus $4.59(S D=4.81)$ for fathers of term infants. Depression levels were not significantly different between VLBW fathers (median $=2$ ) and term fathers (median $=3$ ) at two to four years postpartum, $U=179, z=-2.08, p=.037$. Table 2 presents median and range PHQ-9 and GAD-7 scores for the two groups.

\section{Social Support}

The mean total ESSI (Mitchell et al., 2003) score for fathers of VLBW infants was $26.2(S D=6.46)$ versus $23.8(S D=6.67)$ for fathers of term infants. Scores for VLBW fathers (median $=28$ ) did not differ significantly from those reported by term fathers (median $=24), U=225, z=-1.26, p=.21$. The relationship between perceived social support at the time of the birth and current PTSS was significant in fathers of VLBW infants $(\tau=-.31, n=$ $26, p=.029$ ), with perceived low levels of social support at the time of the birth and infant's hospitalisation associated with higher PTSS at two to four years postpartum. The 
relationships between perceived social support and depression levels, and between perceived social support and anxiety levels were not significant, $\tau=-.19, n=26, p=.28$ and $\tau=-.084$, $n=26, p=.57$. Table 2 presents median and range ESSI scores for the two groups.

\section{Comparison with Mothers' Data}

After combining the paternal data from the present study with the maternal data from Åhlund et al.(2009), the total VLBW group comprised 50 parents (24 mothers and 26 fathers) and the total term group comprised 35 parents (13 mothers and 22 fathers). Table 2 presents the comparative baseline data and IES-R total and sub-scores for both cohorts.

Overall, respondent fathers of VLBW infants had lower PTSS levels at two to four years postpartum (median score $=13$ ) than did mothers of VLBW infants at two to three years postpartum (median score $=25$ ), however, this difference was not statistically significant $(U=223, z=-1.73, p=.085)$. Mothers had higher scores than fathers for the IES$\mathrm{R}$ intrusion subscale (medians: 12 vs. 7 ), $U=199, z=-2.21, p=.027$, but there were no differences in their IES-R avoidance and hyperarousal subscale scores. For parents of termborn infants, reported PTSS was similarly low in mothers at two to three years versus fathers at two to four years (both median $=0$ ), $U=137, z=-.22, p=.84$, and there were no differences in their hyperarousal, intrusion, and avoidance subscale scores (Table 3). There were no significant differences between mothers and fathers in their infants' birth weight, gestational age, and length of hospital stay.

\section{Discussion}

Our study shows that fathers of VLBW infants reported significantly higher levels of PTSS than fathers of term-born infants as late as two to four years postpartum. To our knowledge, ours is the first quantitative study to investigate fathers of VLBW infants at two to four years postpartum and make a direct comparison with a similar cohort of mothers. We 
also found that levels of PTSS in fathers of VLBW infants at two to four years postpartum were similarly high as those previously reported in mothers of VLBW infants at two to three

years postpartum (Åhlund et al., 2009), though the overall trend was towards lower levels of PTSS in the fathers. This is consistent with another study which showed a PTSS reaction in both mothers and fathers but higher scores were observed in mothers compared with fathers (Ionio et al., 2016).

The lack of significant difference between the two groups of fathers with respect to anxiety and depression levels could be because measures designed to assess anxiety and depression can be affected by scores on items that might be associated with having a young infant, such has feeling tired and difficulty relaxing. Research has suggested that the difference between depression and anxiety symptoms in parents of NICU infants and term infants is no longer significant at nine months postpartum (Carter, Mulder, Frampton, \& Darlow, 2007). Infant demographic variables such as birth weight and length of stay in intensive, special and high dependence care, which could be considered an indirect measure of infant illness severity, were not associated with levels of PTSS in fathers of VLBW infants. This is consistent with some previous research (Mackley, Locke, Spear, \& Joseph, 2010), although other research has suggested poor conditions at birth are linked to parents' stress levels (Ionio et al., 2016) and a more likely presence of PTSD for fathers of the most premature infants (Koliouli et al., 2016).

\section{Strengths and Limitations}

PTSS in fathers of VLBW infants has received limited attention in the literature. Ours is the first study to observe longer-term impacts of PTSS reactions in these fathers. We directly invited fathers to participate, rather than inviting them via mothers, thus avoiding a significant limitation of potential bias of many previous studies involving fathers, where 
mothers were the 'gatekeepers' of fathers' research participation (Costigan \& Cox, 2001). An additional strength was our almost-complete control matched group of fathers of termborn infants followed up at the same postnatal age.

Our study had some limitations. Despite best recruitment efforts there were low response rates for consent to contact with questionnaires $(15.1 \%$ for the VLBW group and $13.3 \%$ for the term group) resulting in a small sample size, impacting generalisability. Due to the pilot nature of this study, there were no reliable effect sizes to calculate a priori power analysis. However, the sample size achieved was close to the estimated requirement. The large standard deviations for all questionnaire measures likely reflect the small sample size. Attempts were made to improve response rates with the use of two follow-up reminder letters, which proved successful with a total of $37.8 \%$ of participants who were sent one or two reminder letters returning their questionnaires. Unfortunately follow-up reminders could only be provided for those who responded to the initial invite and consented to the chief investigator contacting them.

Generalisability was also affected by the homogenous sample. The majority of participants were white British (89.6\%) and all were in employment. The methodology meant that it was unlikely that fathers in separated families would have participated. It was not possible to obtain demographic data for non-responding fathers and thus not possible to determine any differences in responders and non-responders in respect of either father or infant demographic characteristics. Responding fathers may have had a larger interest in participating in the study due to increased levels of PTSS symptoms and it is also possible that these fathers had a higher susceptibility for developing PTSS due to prior predictive factors (e.g. previous trauma experience). Alternatively, non-responding fathers may have been experiencing higher levels of PTSS and therefore wanted to avoid thinking about the hospital experience. Avoidance of triggering stimuli is a common symptom of PTSD. 
Self-report measures made it unclear as to whether responses of fathers of VLBW infants on the IES-R pertain specifically to traumatic experiences of the birth of their infant or to traumatic experiences of having their child cared for in the NICU. Evidence would suggest that a cumulative effect of both events caused the elevated levels of PTSS in this group, future research using qualitative methodology could improve the understanding of this relationship which could help to develop more specific interventions for fathers. Self-report using recall for the ESSI to think back to the social support fathers received at the time of the birth of their child two to four years earlier may also have introduced some bias. Use of a historical mother cohort for comparison purposes with our more recent cohort of fathers necessitates some caution in interpretation of findings due to potential bias introduction, despite similarities in methodology. However, the cohorts were from the same region and similar demographic characteristics were observed in the two samples.

Finally, this study was unable to account for past or ongoing medical or psychiatric problems for both fathers and their infants, both of which may have impacted PTSS levels. Future research should consider these and any influence of other stressful/traumatic life events.

\section{Clinical Implications}

Recent evidence suggests a 'screen and treat' approach is valuable for groups at risk for PTSD (Brewin et al., 2008). The reliability shown in the present study of the IES-R indicates it can be used as an appropriate measure for routine clinical screening of fathers of VLBW infants. However, infant's medical severity may not identify fathers of VLBW infants who are at risk of developing PTSD due to the lack of association between variables indirectly measuring this and reported PTSS levels and thus screening for all may prove beneficial. Shaw et al. (2013) have also argued for universal screening of parents rather than 
profiling those at potential high risk because of their infant's medical severity. Within the NICU it has been recommended that screening is completed during hospital admission and at follow-up clinics (Hynan et al., 2013), the follow-up clinic used for recruitment in this study proved a useful way of recruiting and is perhaps an avenue to utilise for increased engagement with screening measures. Screening at follow-up clinics could be particularly important for fathers who may have a delayed PTSS response (Shaw et al., 2009). However, it is important for services to consider support offers prior to screening, if support is not available then screening should not be carried out (Hynan et al., 2013).

Hynan et al. (2015) set out clear recommendations in their paper for mental health support within NICUs including; having a social worker and psychologist on the unit, peerto-peer support, groups for parents and rigorous screening and referral processes for emotional support. This should be happening within the context of normalising and validating emotional distress for parents on the NICU and supporting NICU staff. There are limited specific interventions for postnatal PTSS in mother or fathers and the results of this study point to a need to consider what intervention should be offered following identification of symptoms through screening. A six-session treatment intervention developed to reduce PTSS, depression and anxiety in parents of premature infants, which included psychoeducation, cognitive restructuring, progressive muscle relaxation, and trauma narrative development, resulted in a greater reduction in trauma and depression symptoms in a group of mothers compared to controls not receiving the intervention (Shaw et al., 2013). However, it cannot be assumed that successful interventions for mothers could be effectively applied to fathers. For example, a parental intervention aimed at reducing stress levels of parents of VLBW was effective for mothers but not but not fathers (Matricardi, Agostino, Fedeli, \& Montirosso, 2013). 
Men are often reluctant to seek help and this can lead to poorer health outcomes (White, 2001). Some research has suggested it is difficult for fathers to find support and get acknowledgment of their distress from professionals with others finding it difficult to talk with family and friends, leaving them feeling isolated when dealing with their trauma (Hinton, Locock, \& Knight, 2014). Increasing the awareness that fathers can suffer from postnatal mental health difficulties as well as mothers could help reduce some of the potential barriers to men seeking help for postnatal PTSS and normalise their reactions. Alternative mediums for accessing help which may appeal more to men, such as the internet, should be considered (Sullivan, Camic, \& Brown, 2015). Additionally, engaging fathers from the beginning, during their partner's pregnancy is key (Spencer, 2014) and could aid greater education and normalisation of mental health difficulties as well as developing relationships with professionals which may aid help-seeking.

\section{Future Research}

Our pilot study findings, coupled and compared with those of Åhlund et al. (2009), justify the need for a definitive study in a large cohort of parents of VLBW infants. Studying both parents concurrently would overcome limitations of using an historical cohort for comparison. A large multicentre study would allow a large sample size and increase sample heterogeneity and generalisability of findings.

A recent qualitative study of fathers who experienced a traumatic birth and met some PTSD criteria suggested that the subjective nature of the perception of the birth was crucial and this may have implications for the development of PTSD symptomology (Etheridge \& Slade, 2017). Recent work has shown that it is feasible and acceptable to fathers to administer multiple research questionnaires when investigating this area (Bagge, Westgate, Few, Clarke, \& Adlam, 2017). The use of mixed methods designs could help to unravel the highlighted complexities with regards to the degree to which PTSS is related to the 
experience of the preterm birth relative to the protracted NICU hospitalisation. In addition this could help elucidate whether there was any impact on trauma reactions if the child was the father's first born or only child and any previous losses.

This study did not examine the course of PTSS development or the experience of trauma over time. Future studies would benefit from longer term follow-up, including baseline measures taken during pregnancy and a few days after the birth, as well as measures of previous trauma experiences and the ongoing health of their child which may result in ongoing or repeat traumatisation. This could help to elucidate particularly risky time periods and thus may identify potential risk factors for appropriate targeted intervention in fathers. PTSS in mothers of extremely premature infants has been shown to be present as late as 16 years postpartum (Zerach, Elsayag, Shefer, \& Gabis, 2015). Future studies need to examine if paternal PTSS has a similar long duration.

\section{Conclusions}

This study shows that some fathers of VLBW infants are suffering with PTSS as late as two to four years after the birth of their VLBW infants, and that as a group their levels of PTSS were significantly higher than in a contemporaneous cohort of fathers of term infants. Fathers of VLBW infants also had similarly high PTSS levels at two to four years postpartum as were present in an historical cohort of mothers of VLBW infants at a similar postpartum age. Increased psychological support is indicated for fathers following the birth of a VLBW infant.

Despite the challenge of including fathers in research, this pilot study highlights the need to continue to involve fathers and the importance of analysing them separately from mothers for effects, rather than the current frequent situation where mothers and fathers results are analysed together, often due to the small sample sizes of fathers. More research is urgently needed into the experience and symptoms of trauma for fathers, the potential wider 
impacts on the family, and how the adverse psychological morbidity suffered by fathers of VLBW infants may best be ameliorated.

\section{References}

Åhlund, S., Clarke, P., Hill, J., \& Thalange, N. K. S. (2009). Post-traumatic stress symptoms in mothers of very low birth weight infants 2-3 years post-partum. Archives of Women's Mental Health, 12(4), 261-264. https://doi.org/10.1007/s00737-009-0067-4

All Party Parliamentary Group for Conception to Age 2: The First 1001 Days. (2015). Building Great Britons. Retrieved from http://www.1001criticaldays.co.uk/ buildinggreatbritonsreport.pdf

American Psychiatric Association. (2013). Diagnostic and Statistical Manual of Mental Disorders (5th ed.). Wasington, DC: Author

Asukai, N., Kato, H., Kawamura, N., Kim, Y., Yamamoto, K., Kishimoto, J., ... NishizonoMaher, A. (2002). Reliability and validity of the Japanese-language version of the Impact of Event Scale-Revised (IES-R-J): Four studies of different traumatic events. The Journal of Nervous and Mental Disease, 190, 175-182.

https://doi.org/10.1097/00005053-200203000-00006

Ayers, S., Wright, D., \& Wells, N. (2007). Symptoms of post-traumatic stress disorder in couples after birth: Association with the couple's relationship and parent-baby bond. Journal of Reproductive and Infant Psychology, 25(1), 40-50. https://doi.org/10.1080/02646830601117175

Bagge, S. R., Westgate, B., Few, K., Clarke, P., \& Adlam, A. (2017). Acceptability and feasibility of collecting psychosocial data from fathers of very low birth weight infants. Journal of Child Health Care, 1-9. https://doi.org/10.1177/1367493517715147 
Binder, W. S., Zeltzer, L. K., Simmons, C. F., Mirocha, J., \& Pandya, A. (2011). The father in the hallway: Posttraumatic stress reactions in fathers of NICU babies. Psychiatric Annals, 41(8), 396-402.

Brewin, C. R., Scragg, P., Robertson, M., Thompson, M., D’Ardenne, P., \& Ehlers, A. (2008). Promoting mental health following the london bombings: A screen and treat approach. Journal of Traumatic Stress, 21, 3-8. https://doi.org/10.1002/jts.20310

British Association of Perinatal Medicine. (2011). Categories of Care 2011. Retrieved from https://www.bapm.org/sites/default/files/files/CatsofcarereportAug11.pdf

Carter, J. D., Mulder, R. T., Frampton, C. M. A., \& Darlow, B. A. (2007). Infants admitted to a neonatal intensive care unit: Parental psychological status at 9 months. Acta Paediatrica, 96, 1286-1289. https://doi.org/10.1111/j.1651-2227.2007.00425.x

Costigan, C. L., \& Cox, M. J. (2001). Fathers' participation in family research: Is there a selfselection bias? Journal of Family Psychology, 15, 706-720. https://doi.org/10.1037/0893-3200.15.4.706

Creamer, M., Bell, R., \& Failla, S. (2003). Psychometric properties of the Impact of Event Scale - Revised. Behaviour Research and Therapy, 41, 1489-1496. https://doi.org/10.1016/j.brat.2003.07.010

Deeney, K., Lohan, M., Parkes, J., \& Spence, D. (2009). Experiences of fathers of babies in intensive care. Paediatric Nursing, 21(1), 45-47. https://doi.org/10.7748/paed2009.02.21.1.45.c6913

Elklit, A., Hartvig, T., \& Christiansen, M. (2007). Psychological sequelae in parents of extreme low and very low birth weight infants. Journal of Clinical Psychology in Medical Settings, 14(3), 238-247. https://doi.org/10.1007/s10880-007-9077-4 
Etheridge, J., \& Slade, P. (2017). "Nothing's actually happened to me.”: The experiences of fathers who found childbirth traumatic. BMC Pregnancy and Childbirth, 17(80), 1-15. https://doi.org/10.1186/s12884-017-1259-y

Ghorbani, M., Dolatian, M., Shams, J., \& Alavi-Majd, H. (2014). Anxiety, post-traumatic stress disorder and social supports among parents of premature and full-term infants. Iranian Red Crescent Medical Journal, 16(3), e13461. https://doi.org/10.5812/ircmj.13461

Grinage, B. D. (2003). Diagnosis and management of post-traumatic stress disorder. American Family Physician, 68(12), 2401-2409. Retrieved from http://jlaroche.com/cqfa/280-BVJ/PTSD_2_Diagnostique.pdf

Hinton, L., Locock, L., \& Knight, M. (2014). Partner experiences of "Near-Miss" events in pregnancy and childbirth in the UK: A qualitative study. PLOS ONE, 9(4), e91735. https://doi.org/10.1371/journal.pone.0091735

Hynan, M. T., Mounts, K. O., \& Vanderbilt, D. L. (2013). Screening parents of high-risk infants for emotional distress: Rationale and recommendations. Journal of Perinatology, 33, 748-753. https://doi.org/10.1038/jp.2013.72

Hynan, M. T., Steinberg, Z., Baker, L., Cicco, R., Geller, P. A., Lassen, S., ... Stuebe, A. (2015). Recommendations for mental health professionals in the NICU. Journal of Perinatology, 35, S14-S18. https://doi.org/10.1038/jp.2015.144

Iles, J., Slade, P., \& Spiby, H. (2011). Posttraumatic stress symptoms and postpartum depression in couples after childbirth: The role of partner support and attachment. Journal of Anxiety Disorders, 25, 520-530. https://doi.org/10.1016/j.janxdis.2010.12.006 
Ionio, C., Colombo, C., Brazzoduro, V., Mascheroni, E., Castoldi, F., \& Lista, G. (2016).

Mothers and fathers in NICU : The impact of preterm birth on parental distress.

Europe's Journal of Psychology, 12(4), 604-621.

https://doi.org/10.5964/ejop.v12i4.1093

Koliouli, F., Gaudron, C. Z., \& Raynaud, J. (2016). Stress, coping, and post-traumatic stress disorder of French fathers of premature infants. Newborn and Infant Nursing Reviews, 16, 110-114. https://doi.org/10.1053/j.nainr.2016.08.003

Kroenke, K., \& Spitzer, R. L. (2002). The PHQ-9 : A New Depression Measure. Psychiatric Annals, 32, 509-515. https://doi.org/170553651

Lefkowitz, D. S., Baxt, C., \& Evans, J. R. (2010). Prevalence and correlates of posttraumatic stress and postpartum depression in parents of infants in the Neonatal Intensive Care Unit (NICU). Journal of Clinical Psychology in Medical Settings, 17(3), 230-237. https://doi.org/10.1007/s10880-010-9202-7

Mackley, A. B., Locke, R. G., Spear, M. L., \& Joseph, R. (2010). Forgotten parent: NICU paternal emotional response. Advances in Neonatal Care, 10(4), 200-203. https://doi.org/10.1097/ANC.0b013e3181e946f0

Matricardi, S., Agostino, R., Fedeli, C., \& Montirosso, R. (2013). Mothers are not fathers: Differences between parents in the reduction of stress levels after a parental intervention in a NICU. Acta Paediatrica, International Journal of Paediatrics, 102(1), 8-14. https://doi.org/10.1111/apa.12058

Matthey, S., Barnett, B., Ungerer, J., \& Waters, B. (2000). Paternal and maternal depressed mood during the transition to parenthood. Journal of Affective Disorders, 60(2), 75-85. https://doi.org/10.1016/S0165-0327(99)00159-7 
Mehler, K., Mainusch, A., Hucklenbruch-Rother, E., Hahn, M., Hunseler, C., \& Kribs, A. (2014). Increased rate of parental postpartum depression and traumatization in moderate and late preterm infants is independent of the infant's motor repertoire. Early Human Development, 90, 797-801. https://doi.org/10.1016/j.earlhumdev.2014.09.008

Mitchell, P. H., Powell, L., Blumenthal, J., Norten, J., Ironson, G., Pitula, C. R., ... Berkman, L. F. (2003). A short social support measure for patients recovering From myocardial infarction: The ENRICHD social support inventory. Journal of Cardiopulmonary Rehabilitation and Prevention, 23(6), 398-403. http://doi.org/10.1097/00008483200311000-00001

Office for National Statistics. (2014). Gestation-specifc infant mortality. Cardiff: Author. Retrieved from http://www.ons.gov.uk/ons/publications/re-reference-tables.html? edition=tcm:77-349394

Paulson, J. F., \& Bazemore, S. D. (2010). Prenatal and postpartum depression in fathers and its association with maternal depression: A meta-analysis. JAMA, 303(19), 1961-1969.

Phares, V., Lopez, E., Fields, S., Kamboukos, D., \& Duhig, A. M. (2005). Are fathers involved in pediatric psychology research and treatment? Journal of Pediatric Psychology, 30(8), 631-643. https://doi.org/10.1093/jpepsy/jsi050

Shaw, R. J., Bernard, R. S., DeBlois, T., Ikuta, L. M., Ginzburg, K., \& Koopman, C. (2009). The relationship between acute stress disorder and posttraumatic stress disorder in the neonatal intensive care unit. Psychosomatics, 50(2), 131-137.

Shaw, R. J., St John, N., Lilo, E. A., Jo, B., Benitz, W., Stevenson, D. K., \& Horwitz, S. M. (2013). Prevention of traumatic stress in mothers with preterm infants: A randomized controlled trial. Pediatrics, 132(4), e886-894. https://doi.org/10.1542/peds.2013-1331 
Spencer, O. (2014). Sad Dad: An exploration of postnatal depression in fathers. Croydon, UK: CPI Group.

Spitzer, R. L., Kroenke, K., Williams, J. B., \& Löwe, B. (2006). A brief measure for assessing generalized anxiety disorder: The GAD-7. Archives of Internal Medicine, 166(10), 1092-1097. https://doi.org/10.1001/archinte.166.10.1092

Stramrood, C. A., Paarlberg, K. M., Huis In 't Veld, E. M., Berger, L. W., Vingerhoets, A. J., Schultz, W. C., \& van Pampus, M. G. (2011). Posttraumatic stress following childbirth in homelike and hospital settings. Journal of Psychosomatic Obstetrics and Gynaecology, 32(2), 88-97. https://doi.org/10.3109/0167482X.2011.569801

Sullivan, L., Camic, P. M., \& Brown, J. S. L. (2015). Masculinity, alexithymia, and fear of intimacy as predictors of UK men's attitudes towards seeking professional psychological help. British Journal of Health Psychology, 20(1), 194-211. https://doi.org/10.1111/bjhp.12089

Weiss, D. S., \& Marmar, C. R. (1997). The Impact of Event Scale-revised. In Assessing psychological trauma and PTSD (pp. 399-411). https://doi.org/10.1007/978-0-38770990-1_10

White, A. (2001). Report on the scoping study on men's Health. London, UK: HMSO.

White, T., Matthey, S., Boyd, K., \& Barnett, B. (2006). Postnatal depression and posttraumatic stress after childbirth: Prevalence, course and co-occurrence. Journal of Reproductive and Infant Psychology, 24(2), 107-120. https://doi.org/10.1080/02646830600643874

Zerach, G., Elsayag, A., Shefer, S., \& Gabis, L. (2015). Long-Term Maternal Stress and Posttraumatic Stress Symptoms Related to Developmental Outcome of Extremely Premature 
Infants, 213, 204-213. https://doi.org/10.1002/smi.2547

\section{Tables}

Table 1.

Baseline characteristics of participating fathers

\begin{tabular}{|c|c|c|}
\hline & $\begin{array}{l}\text { Very Low Birth Weight } \\
\qquad(n=26)\end{array}$ & $\begin{array}{c}\text { Term } \\
(n=21)^{1}\end{array}$ \\
\hline \multicolumn{3}{|l|}{ Ethnicity } \\
\hline White British & $22(85)$ & $21(96)$ \\
\hline White Irish & $1(4)$ & $0(0)$ \\
\hline White European & $1(4)$ & $0(0)$ \\
\hline Black Other & $1(4)$ & $0(0)$ \\
\hline Asian British & $1(4)$ & $0(0)$ \\
\hline \multicolumn{3}{|l|}{ Age Group } \\
\hline $25-29$ & $0(0)$ & $2(9)$ \\
\hline $30-34$ & $8(31)$ & $6(27)$ \\
\hline $35-39$ & $9(35)$ & $6(27)$ \\
\hline $40-44$ & $5(19)$ & $4(18)$ \\
\hline $45-49$ & $3(12)$ & $2(10)$ \\
\hline $50+$ & $1(4)$ & $1(5)$ \\
\hline
\end{tabular}

Data are $n(\%) .{ }^{\mathrm{I}}$ Demographic data from one participant were missing (questionnaire not returned). 
Table 2.

Mann-Whitney Test Statistics for Anxiety, Depression and Social Support Data

\begin{tabular}{|c|c|c|c|c|c|}
\hline & \multicolumn{2}{|c|}{ Median Scores $(I Q R)$} & $U$ & $z$ & $p$ \\
\hline & $\begin{array}{l}\text { Very Low Birth Weight } \\
\qquad(n=26)\end{array}$ & $\begin{array}{c}\text { Term } \\
(n=22)\end{array}$ & & & \\
\hline GAD- $7^{1}$ & $2(0-6)$ & $4(2-6)$ & 228 & -1.22 & .23 \\
\hline PHQ-9² & $2(0-4)$ & $3(1-5)$ & 179 & -2.08 & .037 \\
\hline $\mathrm{ESSI}^{3}$ & $28(22-32)$ & $24(19-30)$ & 225 & -1.26 & .21 \\
\hline
\end{tabular}

${ }^{\mathrm{I}}$ GAD-7 = Generalised Anxiety Disorder Seven-Item Scale. ${ }^{2}$ PHQ-9 = Patient Health Questionnaire-9 Depression Scale. ${ }^{3}$ ESSI $=$ ENRICHD Social Support Inventory. 
Table 3.

Mann-Whitney Test Statistics for Father-Mother Comparisons

\begin{tabular}{|c|c|c|c|c|c|}
\hline & \multicolumn{2}{|c|}{ Median $(I Q R)$ Scores } & \multirow[t]{3}{*}{$U$} & \multirow[t]{3}{*}{$z$} & \multirow[t]{4}{*}{$p$} \\
\hline & \multicolumn{2}{|c|}{ Parents of Very Low Birth Weight } & & & \\
\hline & \multicolumn{2}{|c|}{ Infants } & & & \\
\hline & Fathers $(n=26)$ & Mothers $(n=24) * *$ & & & \\
\hline IES-R† Total Scale & $13(24)$ & $25(24)$ & 223.0 & -1.73 & .085 \\
\hline IES-R Intrusion Scale & $7(8.7)$ & $12(11.8)$ & $198.5 *$ & -2.21 & .027 \\
\hline IES-R Avoidance Scale & $5(11.8)$ & $8(8.8)$ & 277.5 & -0.67 & .51 \\
\hline IES-R Hyperarousal Scale & $1(4.75)$ & $3(6)$ & 224.0 & -1.75 & .081 \\
\hline Birth Weight (grams) & $1060(582)$ & $1120(412)$ & 307.0 & -0.10 & .93 \\
\hline Gestational Age (weeks) & $28(3.4)$ & $30(5)$ & 221.5 & -1.76 & .08 \\
\hline Days Infant in Hospital & $45(45)$ & $46(35)$ & 311.5 & -0.01 & .10 \\
\hline Days Infant in Intensive & $6.5(19)$ & $2.5(6)$ & $175.5^{*}$ & -2.67 & .007 \\
\hline Care & & & & & \\
\hline
\end{tabular}




\begin{tabular}{|c|c|c|c|c|c|}
\hline Days Infant in High & $15(17)$ & $1.5(10)$ & $177.0^{*}$ & -2.66 & .007 \\
\hline \multicolumn{6}{|l|}{ Dependency Care } \\
\hline Days Infant in Special & $21(30)$ & $38(26)$ & $156.5^{*}$ & -3.02 & .002 \\
\hline \multicolumn{6}{|l|}{ Care } \\
\hline \multicolumn{6}{|c|}{ Parents of Term Infants } \\
\hline & Fathers $(n=22)$ & Mothers $(n=13)^{* *}$ & & & \\
\hline IES-R ${ }^{1}$ Total Scale & $1(3)$ & $0(3)$ & 137.0 & -0.22 & .84 \\
\hline IES-R Intrusion Scale & $0(2)$ & $0(1)$ & 131.0 & -0.46 & .67 \\
\hline IES-R Avoidance Scale & $0(0)$ & $0(0.5)$ & 138.5 & -0.22 & .99 \\
\hline IES-R Hyperarousal Scale & $0(0)$ & $0(1)$ & 129.5 & -0.63 & .65 \\
\hline Birth Weight (grams) & $3593(610)$ & $3450(1050)$ & 96.0 & -0.96 & .36 \\
\hline Gestational Age (weeks) & $40(1.1)$ & $40(3.4)$ & 117.5 & -0.13 & .90 \\
\hline
\end{tabular}

*Significant at the .05 level (2-tailed). ${ }^{1}$ IES-R = Impact of Events Scale Revised.

***Maternal data from Åhlund et al.(Ahlund et al., 2009), with kind permission. 
Figure 1. Recruitment Flow Diagram

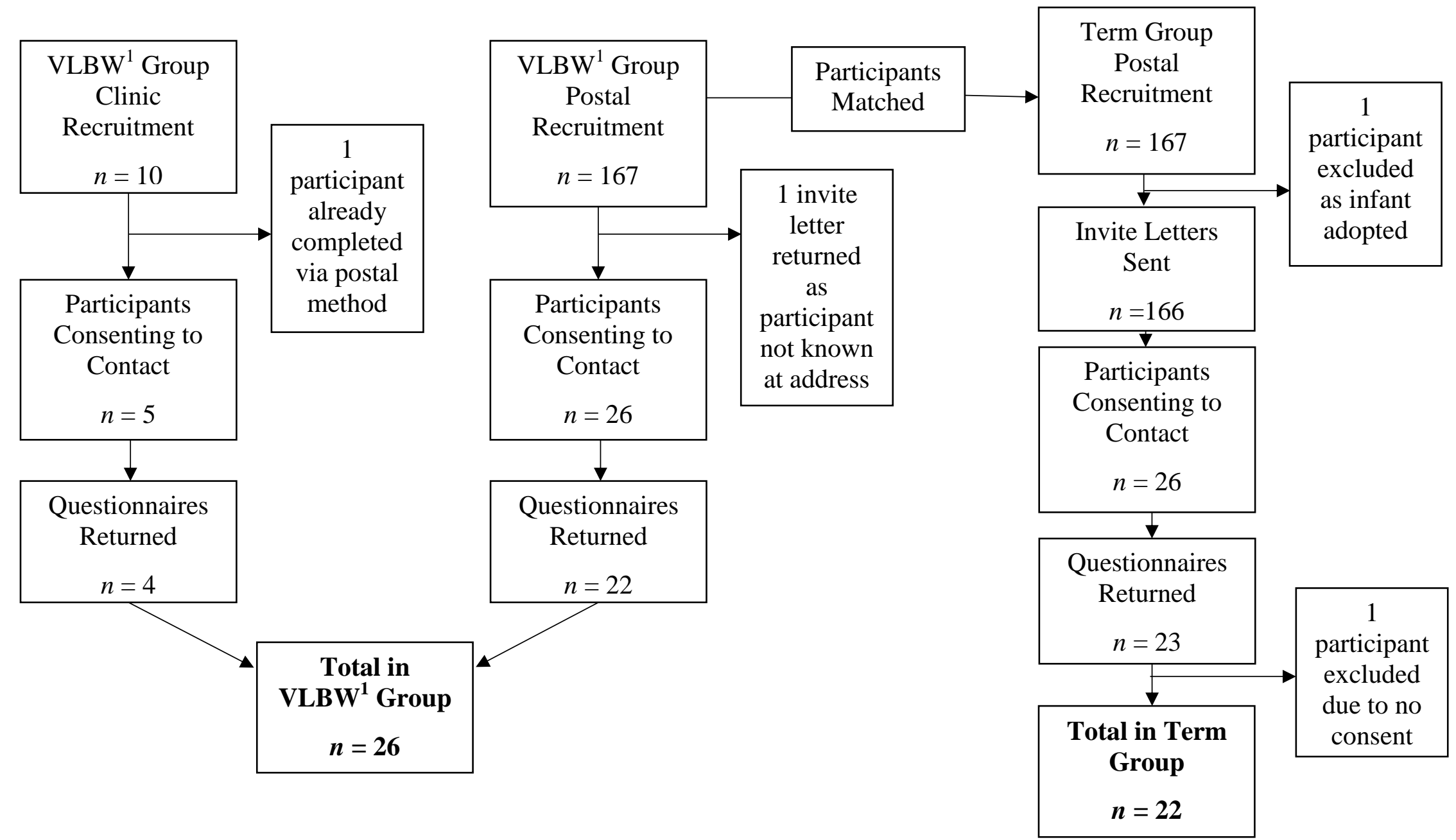

${ }^{1}$ VLBW $=$ Very Low Birth Weight 